\title{
Posición COMPETITIVA Y MODELO PRODUCTIVO EN el Perú
}

\author{
(1) José Porlles Loarte \\ (2) Carlos Quispe Atúncar \\ (3) Máximo Leyva Caballero \\ (4) Manuel Bejar Ramos
}

\begin{abstract}
RESUMEN
Enelmarcodelacompetitividadmundial, el desempeño del Penútiende hacia un deterioro preocupante, másaún, cuandonosevislumbranla construccióndenuevasfuerzas pararevertirel proceso, manteniendo un modelo económicoy, por lotanto, un modelo productivo que, en la medida quesigamosdependiendo delosrecursos naturales, no garantizala produccióny exportación de productos conalto valor agregado. Parael análisis, sehantomadoen consideración losúltimos informes delWorldEconomic Forum(WEF)sobre losíndicesdecompetitividad, laevolucióndela economíamundial, regionalydel país.

Palabras Clave: Competitividad, desempeño,
\end{abstract} tecnología, crecimiento, modelo productivo.

COMPETITIVE POSITION ANDPRODUCTIVE MODEL IN PERU

ABSTRACT

In the world competitivenessframework, the performance of Peru goes towarda worrying deterioration. Even more, when the construction of newforcesto revertthe processisnotbecoming visible, maintaininganeconomicmodeland, therefore, a productive model that does not guaranteethe production and export of products with high aggregatevalue, to the extent thatwe continue depending on the natural resources. For purpose of analysis, the lastreports of the World Economic Fonum(WEF)onthecompeitiveness indexand the evolution of the country, regional and world economy have been taken into consideration.

Key Words: Competitiveness, performance technology, growth, productive model.

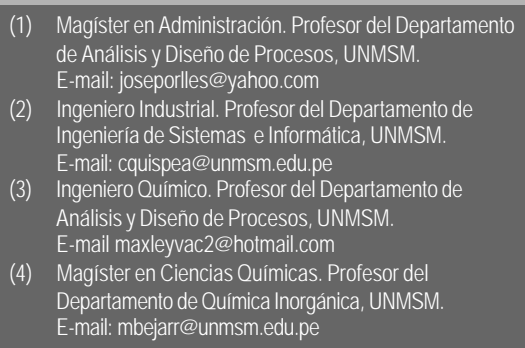

1) Magister en Administración. Profesor del Departamento de Análisis y Diseño de Procesos, UNMSM.

Ingeniero Industrial. Profesor del Departamento de Ingeniería de Sistemas e Informática, UNMSM. E-mail: cquispea@unmsm.edu.pe

Ingeniero Químico. Profesor del Departamento de

E-mail maxleyvac2@hotmail.com

Departamento de Química Inorgánica, UNMSI E-mail: mbejarr@unmsm.edupe

\section{INTRODUCCIÓN}

En el contexto de competencia actual y a la luz de la más reciente publicación del Reporte Global de Competitividad, publicado por el Foro Económico Mundial para el periodo 2005/2006 (13), se ha considerado relevante desarrollar este trabajo, en la medida que los países y empresas, con más posibilidades de éxito en el largo plazo, son aquellos que configuran un sólido nivel de competitividad y que, a su vez, grafican: Desarrollo tecnológico, eficiencia, eficacia, rentabilidad y capacidad de generación de riqueza. Hoy la competitividad se ha convertido en una necesidad ineludible para sostener el crecimiento empresarial y el de las naciones.

En esta línea de pensamiento, el presente trabajo comprende los siguientes objetivos:

1. Visualizar la posición competitiva del Perú en el contexto mundial y latinoamericano.

2. Proporcionar una prospección resumida de la producción nacional y apreciar un siglo del crecimiento del Perú y algunos países de América Latina.

3. Apreciar los impulsos que afectan la evolución del crecimiento, la productividad y la inversión en el Perú.

4. Sugerir algunas medidas para revertir las situaciones negativas.

\section{CONCEPTUALIZACIÓN DE LA COMPETITIVIDAD}

La competitividad puede definirse como la capacidad que tienen los países, regiones y empresas para crecer en forma sostenida en el largo plazo, en un contexto de competencia globalizada.

\section{EL REPORTE GLOBAL DE COMPETITIVIDAD}

El Foro Económico Mundial, una entidad independiente con sede en Ginebra, en alianza con la Universidad de Harvard estructuran y publican dicho reporte con los siguientes índices:

\section{Ranking de Competitividad para el Crecimiento (ICC)}

Se fundamenta en la medición de tres factores que son pilares y contribuyen a crear las condiciones para un crecimiento sostenido: El entorno macroeconómico, la calidad de las instituciones públicas y la tecnología. Este último factor se considera trascendental para el crecimiento a largo plazo, en cuanto se estima que está muy relacionado con la generación del PBI. En este punto, cabe anotar que el Perú es uno de los países de la región que invierte muy poco en investigación y desarrollo. 


\section{Ranking de Competitividad Empresarial (ICE)}

Se fundamenta en la medición de los factores que sostienen la productividad actual, reflejando los fundamentos microeconómicos sobre la sofisticación de la empresa, en cuanto a los modelos de estrategias y en cuanto la calidad del entorno de los negocios.

En síntesis, el Indice Global de Competitividad mide el crecimiento potencial de una economía en un horizonte de cinco a diez años; es decir, mide la capacidad de los países para alcanzar crecimiento económico sostenido e identifica los factores por los cuales unos países tienen más éxito para mejorar el nivel de vida de su población. Es decir, evalúa las fortalezas y debilidades de los países en su inserción en la economía global. De allí la importancia de este reporte, por cuanto constituye un instrumento de toma de decisiones de inversión para los inversionistas en el mundo.

Metodología de medición y factores claves para un crecimiento sostenido El ICC se fundamenta en tres ideas centrales:

- Medición de tres factores claves que contribuyen a crear las condiciones para un crecimiento sostenido: El entorno macroeconómico, la calidad de las instituciones públicas y la tecnología, que constituyen los basamentos sobre los que descansa el proceso de crecimiento económico.

- El factor tecnológico como otro factor clave, en particular a largo plazo, dado que se considera altamente correlacionado a la generación del PBI.

- Distinción entre los países que desarrollan decididamente el concepto tecnológico y aquellos que no lo hacen, como es el caso del Perú.

Cuadro 1. Evolución de los ńdices de competitividad mundial

\begin{tabular}{|c|c|c|c|c|c|c|c|c|c|}
\hline \multicolumn{5}{|c|}{$\begin{array}{l}\text { Indice de Competitividad } \\
\text { para el Crecimiento (ICC) }\end{array}$} & \multicolumn{5}{|c|}{$\begin{array}{l}\text { Indice de Competitividad } \\
\text { Empresarial (ICE) }\end{array}$} \\
\hline País & 2002 & 2003 & \begin{tabular}{|l|}
2004 \\
\end{tabular} & 2005 & País & 2002 & 2003 & 2004 & 2005 \\
\hline Finlandia & 1 & 1 & 1 & 1 & USA & 1 & 2 & 1 & 1 \\
\hline USA & 2 & 2 & 2 & 2 & Finlandia & 2 & 1 & 2 & 2 \\
\hline Japón & 13 & 11 & 9 & 12 & Alemania & 4 & 5 & 3 & 3 \\
\hline Alemania & 14 & 13 & 13 & 15 & \begin{tabular}{|l} 
Japón \\
\end{tabular} & 11 & 13 & 8 & 8 \\
\hline Korea & 21 & 18 & 29 & 17 & Korea & 23 & 23 & 24 & 24 \\
\hline Chile & 26 & 28 & 22 & 23 & Chile & 31 & 32 & 29 & 29 \\
\hline China & 42 & 44 & 46 & 49 & India & 37 & 37 & 30 & 31 \\
\hline Italia & 39 & 41 & 47 & 47 & Italia & 25 & 24 & 34 & 38 \\
\hline Mexico & 45 & 47 & 48 & 55 & Brazil & 33 & 34 & 38 & 49 \\
\hline India & 53 & 56 & 55 & 50 & China & 38 & 46 & 47 & 57 \\
\hline Brazil & 52 & 54 & 57 & 65 & Mexico & 55 & 48 & 55 & 60 \\
\hline Perú & 54 & 57 & 67 & 68 & Perú & 66 & 81 & 76 & 81 \\
\hline tal & 80 & 102 & 104 & 117 & Total & 80 & 101 & 103 & 116 \\
\hline
\end{tabular}

Fuente: Reportes del WEF. El último que corresponde al periodo 20052006 fue publicado el 28 de Septiembre del 2005

\section{POSICIÓN COMPETITIVA DEL PERU}

Reporte de competitividad mundial 2002/2005

- ¿No es preocupante la pérdida de nuestra posición competitiva? América Latina retrocede. Sólo Chile destaca como el país más competitivo de la región. Perú muestra retroceso como tendencia (véase Cuadro 1).

- En el índice de competitividad para el crecimiento (ICC) el Perú está en promedio en el tercio menos competitivo.

- El Perú presenta uno de los niveles más bajos de competitividad empresarial. Estamos en promedio en el quinto menos competitivo.

- ¿Quién nos gana en América Latina y El Caribe (ALC)? El Salvador, República Dominicana, ni qué decir de Chile, Brasil y México.

Michael Porter, experto sobre estrategia y competitividad, en una entrevista con el Wall Street Journal (2), revela que ha habido una gran decepción en el desempeño de la mayoría de los países de ALC, añadiendo que en ALC hubo demasiada concentración en las reformas macroeconómicas y en la infraestructura física, sin prestar suficiente atención a la parte microeconómica, como la competitividad y las acciones conducentes a mejorar la eficiencia. En el caso del Perú, anota "... sigue dependiendo de los recursos naturales".

Bajo este enfoque, Porter afirma: Es el turno de las empresas. Que las reformas macro son muy dolorosas; requieren grandes sacrificios, pero no generan, por sí solas, economías más productivas. Que la productividad depende de las firmas, de su capacidad para competir en niveles más altos de eficiencia. Sólo hay una manera de crear riqueza y ésta es a través de las empresas, cuando pueden ser eficientes y productivas en bienes y servicios y venderlos al mercado internacional.

\section{SITUACIÓN DEL CONTEXTO MUNDIAL Y DE LAS REGIONES COMO AMÉRICA LATINA Y EL CARIBE (ALC)}

¿Cuáles son las perspectivas? Con un repunte del crecimiento mundial en el 2004 (11) se registra una tendencia sostenible en el 2005 (Figura 1.). Si bien en el futuro se prevén riesgos elevados, el FMI parte de la hipótesis de un crecimiento mundial vigoroso y continuo que, según las proyecciones, se mantendrá por encima de la tendencia actual (Figura 2). En esta tendencia, se prevé un crecimiento de ALC y del Perú (5). ¿Pero es suficiente para acortar la brecha entre los países industrializados y los países en desarrollo, como es el caso de ALC? 


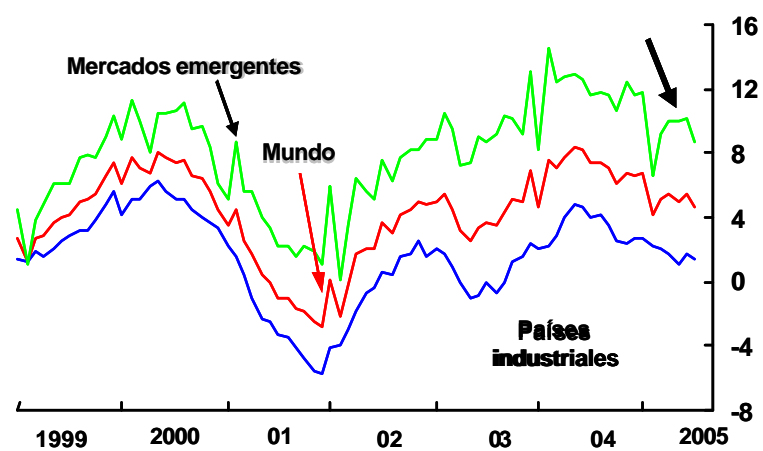

Figura 1. Repùnte de la producción mundial Fuente: FMI, octubre 2005.

Dentro de esta perspectiva general, existen marcadas divergencias entre regiones, siendo Estados Unidos y China los países que se están expandiendo más rápidamente (véase Figura 2).

En efecto, dentro del mismo bloque de economías en desarrollo, ALC no muestra su mejor desempeño. Las economías denominadas "Tigres del Asia”: India y China proyectan una tasa de crecimiento en promedio del $7 \%$ que sí avizora un crecimiento sostenible, en el largo plazo, que les permitiría acercarse cada vez más a las economías industriales (Figura 3).

Desde la perspectiva de las exportaciones mundiales, también se aprecia desigualdad en la velocidad de crecimiento comercial. Pese a los recientes avances, ALC sigue siendo una región relativamente cerrada al comercio internacional, en comparación con otras regiones más dinámicas.

Efectivamente, resulta preocupante que las proyecciones de la exportación de los países de ALC, como proporción de su PBI, indiquen que se registrará un

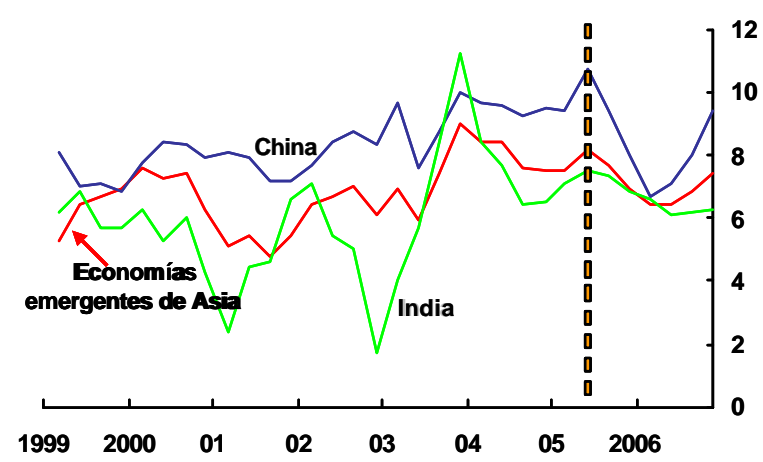

Figura 3. Crecimiento en las economías emergentes de Asia (\% PBI real)

Fuente: FMI, octubre 2005

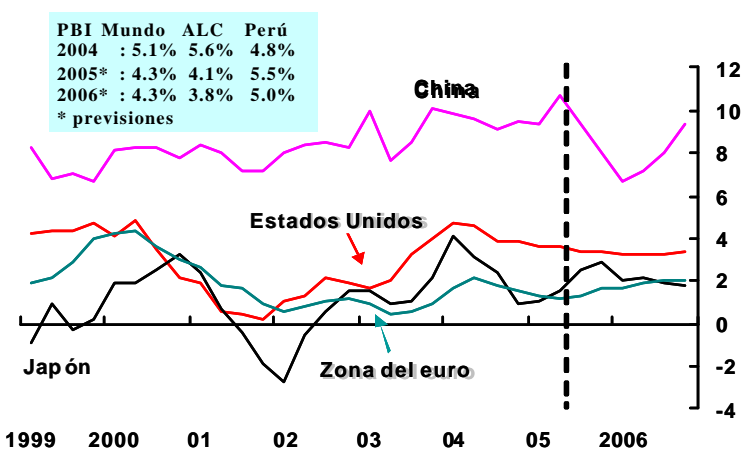

Figura 2. Crecimiento del PBI real Fuente: FMI, octubre 2005

descenso, mientras que en otras regiones, se prevé una mayor integración a los mercados internacionales (Figura 4).

En la región de ALC el crecimiento se está moderando tras alcanzar, en el 2004, el nivel más alto de los últimos 24 años (Figura 5). No obstante, las tasas de crecimiento previstas en un $4,1 \%$ en el 2005 y un $3,8 \%$ en el 2006 , siguen siendo muy superiores a la media histórica $(2,6 \%)$.

Se debe anotar que el vigoroso crecimiento de China y los "Tigres de Asia" han sido destino de ciertas exportaciones básicas de ALC, como el cobre y la soja, con los cuales China representa la quinta parte del consumo mundial. Además, este país ha provocado un aumento de los precios de estos productos, beneficiando a exportadores como Argentina, Brasil, Chile y Perú. Pero aún es insuficiente la recuperación de ALC para impulsar progreso sostenido a la gran población pobre con que cuenta la región. ALC crece a un ritmo menor que otras regiones (Figura 6).

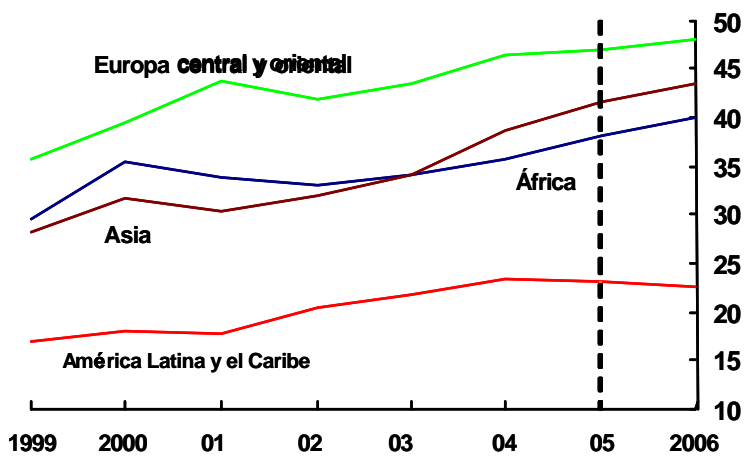

Figura 4. Exportaciones mundiales de bienes y servicios $(\% \mathrm{PBI})$

Fuente: FMI, octubre 2005 


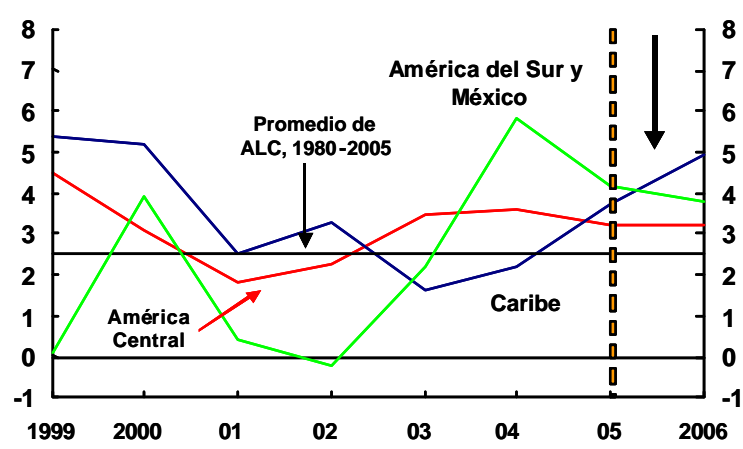

Figura 5. Crecimiento del PBI en ALC (\% PBI real) Fuente: FMI, octubre 2005.

Si bien se han logrado importantes avances de política económica, aún falta mucho por hacer. Una medida fundamental para el futuro será consolidar las bases para lograr un aumento sostenido del crecimiento, para cerrar su brecha, con respecto a otras regiones de mercados emergentes dinámicos.

Al analizar el ingreso per cápita, se visualiza el crecimiento de la brecha entre ALC y las economías desarrolladas y de la región del sudeste asiático (Figura 7). ALC no ha logrado estar a la par con la región del sudeste asiático. En la década del 80 , decreció en promedio cerca del $1 \%$ anual, en tanto que los asiáticos crecieron al $4 \%$ promedio anual. En la década del 90, ALC se recuperó creciendo sólo al 1,5\% promedio anual, pero la región asiática creció más del doble: $3,2 \%$.

Como resultado, la pobreza no está disminuyendo. El Perú cuenta con una población pobre (ingreso personal menor de 2 dólares por día/año) del 52\% (4), compartiendo lugares similares con países como Indonesia, Kenya, Nepal, entre otros (8).

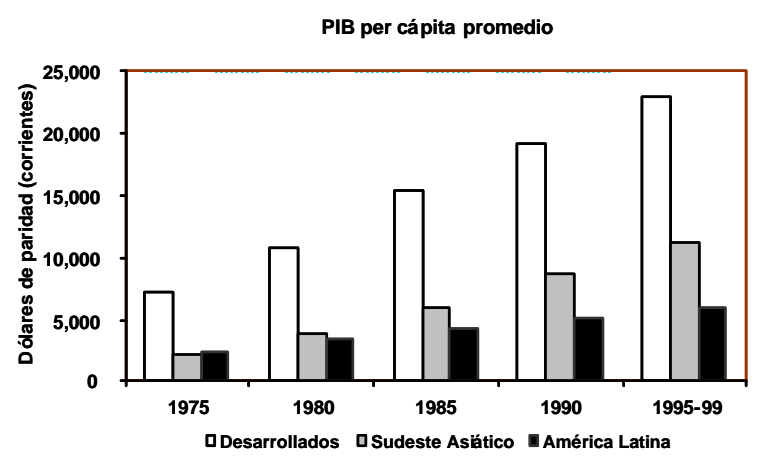

Figura 7. Crecimiento de las brechas de ingreso en los países más desarrollados Fuente: Banco Mundial, 2000.

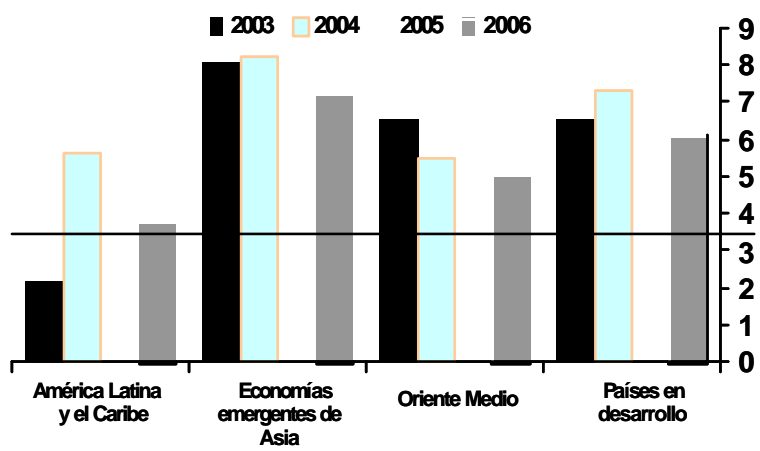

Figura 6. Comparación de crecimiento del PBI en las regiones Fuente: FMI, octubre 2005.

\section{¿ALC es un polo de atracción para la in-} versión extranjera directa?

Según el reciente reporte de la UNCTAD, en el 2004, las IED tuvieron un modesto incremento del $2 \%$ con relación al 2003 (12). Estados Unidos, Reino Unido y China lideran la recepción de las IED en el mundo. Los países en desarrollo han sido los grandes beneficiados, ya que su recepción de las IED creció en un $40 \%$, con relación al año anterior. Y dentro de este gran grupo ALC repunta, dado que después de cuatro años de descenso continuo, las IED crecieron $44 \%$ frente a lo invertido en el 2003. Sin embargo, dentro de ALC, los países deben competir entre ellos para atraer los flujos de IED destinados a la región. Las cifras son reveladoras (Cuadro 2), el Perú no está ganando en esa competencia.

\section{DESEMPEÑO ECONOMICO GLOBAL DEL PERÚ}

Es importante visualizar el desempeño del Perú en un largo periodo de tiempo y en el contexto latinoamericano. Como se aprecia en la Figura 8, el Perú

Cuadro 2. Evolución de la Inversión Extranjera Directa (IED)

\begin{tabular}{|c|c|c|c|c|c|}
\hline \multicolumn{3}{|c|}{$\begin{array}{l}\text { LAS INVERSIONES EN EL MUNDO } \\
\text { (IED en Miles de Millones de } \$ \text { ) }\end{array}$} & \multicolumn{3}{|c|}{$\begin{array}{l}\text { INVERSIONES EXTRANJERAS } \\
\text { Grandes Diferencias en AL }\end{array}$} \\
\hline & 2003 & 2004 & \multirow{2}{*}{\begin{tabular}{|l|} 
Miles de \\
Millones \$
\end{tabular}} & 2003 & 2004 \\
\hline & & & & & \\
\hline \multirow{4}{*}{$\begin{array}{l}\text { Total Mundial } \\
\text { Africa } \\
\text { Europa Sureste } \\
\text { y CIS }\end{array}$} & 632 & 648 & \multirow{9}{*}{$\begin{array}{l}\text { Total AL } \\
\text { Brasil } \\
\text { Mexico } \\
\text { Chile } \\
\text { Argentina } \\
\text { Bermuda } \\
\text { Islas Caimán } \\
\text { Colombia } \\
\text { Perú }\end{array}$} & 47,1 & 67,5 \\
\hline & & & & & 18,2 \\
\hline & & & & & 16,6 \\
\hline & & & & & 7,6 \\
\hline ALC & 47 & 68 & & & 4,3 \\
\hline \multirow[t]{2}{*}{ Asia y Oceanía } & & & & & 3,8 \\
\hline & & & & & 3,0 \\
\hline & & & & & 2,7 \\
\hline esarrollados & & 380 & & 1,3 & 1,8 \\
\hline
\end{tabular}

Fuente: UNTACD, «World Invesment Report 2005», publicado el 29 de Setiembre 2005. 


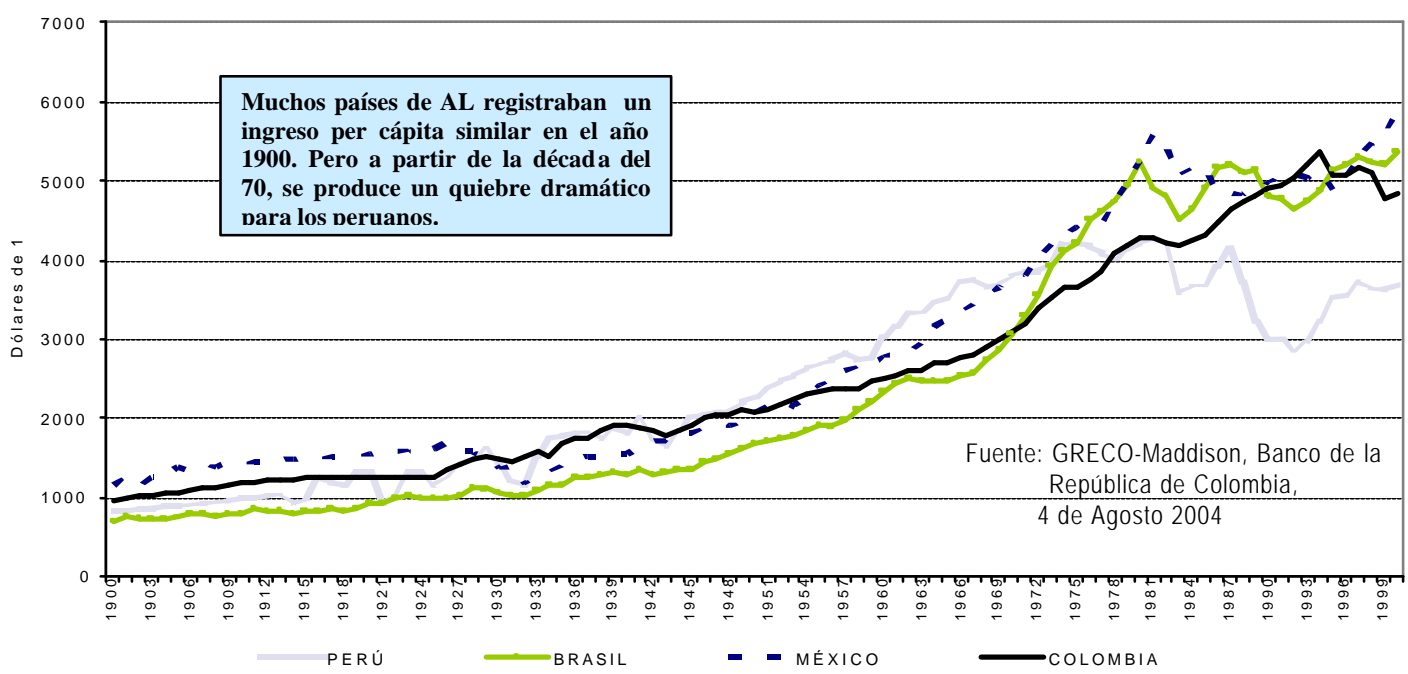

Figura 8. Un siglo de crecimiento económico:1900-2000. Niveles de PBI per cápita, datos de 1990

es el gran perdedor en los últimos cien años en el ámbito del crecimiento económico frente a países como Colombia, Brasil. México, Chile, Argentina y Venezuela.

\section{¿Qué ha sucedido con nuestra capacidad de generación de riqueza?}

El nivel de riqueza nacional actual es similar a los años 70: 2300 dólares/persona/año (Figura 9). Usualmente decimos que se ha perdido sólo tres décadas, pero en realidad, el costo de oportunidad ha sido demasiado alto para la Nación.

El esfuerzo a desplegar es grande para recuperar las décadas perdidas, más aún, cuando nuestra economía representa sólo entre el 0,2-0,3\% del PBI mundial (3).

\section{EL MODELO DE LA ACTIVIDAD ECONÓMI- CA Y PRODUCTIVA DEL PERÚ}

\section{Radiografía de la producción nacional} ONUDI (6), considera que el desarrollo productivo es la fuerza motriz de la aplicación de nuevas tecnologías a la producción y es la fuente y el agente de difusión má importante de la innovación tecnológica.

En el mundo y en los países desarrollados, el peso de la manufactura en el PBI es del $22 \%$; en tanto que los países asiáticos más dinámicos tienden a un valor encima del $25 \%$. En el Perú, el sector manufactura ha representado, en promedio, sólo el $16 \%$ de la economía en los últimos 20 años.
Si bien la economía nacional y el producto no primarios han crecido a una tasa en promedio del $4,5 \%$ y $4,0 \%$, respectivamente, en el periodo 1993-2004, éstos han estado por debajo de la velocidad de crecimiento del sector manufacturero en otras regiones durante el periodo 1980-2000; como señala la ONUDI: En Asia del Sur (6,5\%) y Asia del Este (9,0\%). La tasa de crecimiento de la producción de productos primarios es mayor que la de productos industriales (Figura 10), impulsando, desde 1998, el crecimiento del PBI nacional.

\section{Estructura de las Exportaciones}

Después de más de 20 años, se produce un quiebre favorable en la balanza comercial, con un crecimien-

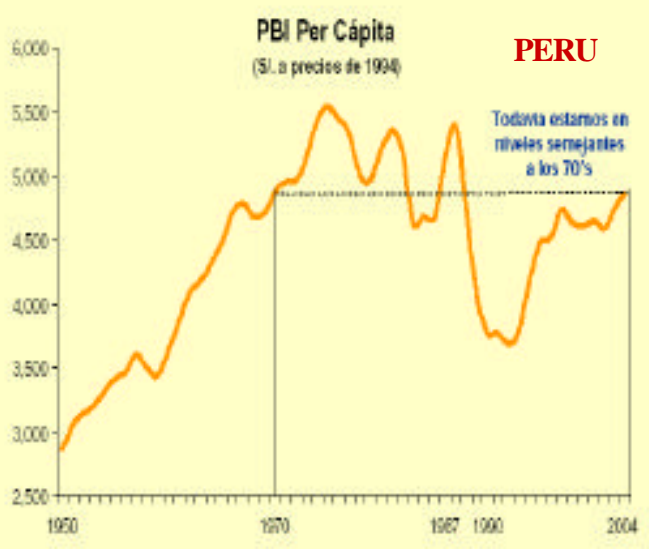

Figura 9. Tendencia del PBI per cápita: 1950-2004 Fuente: INEI, 2005. 


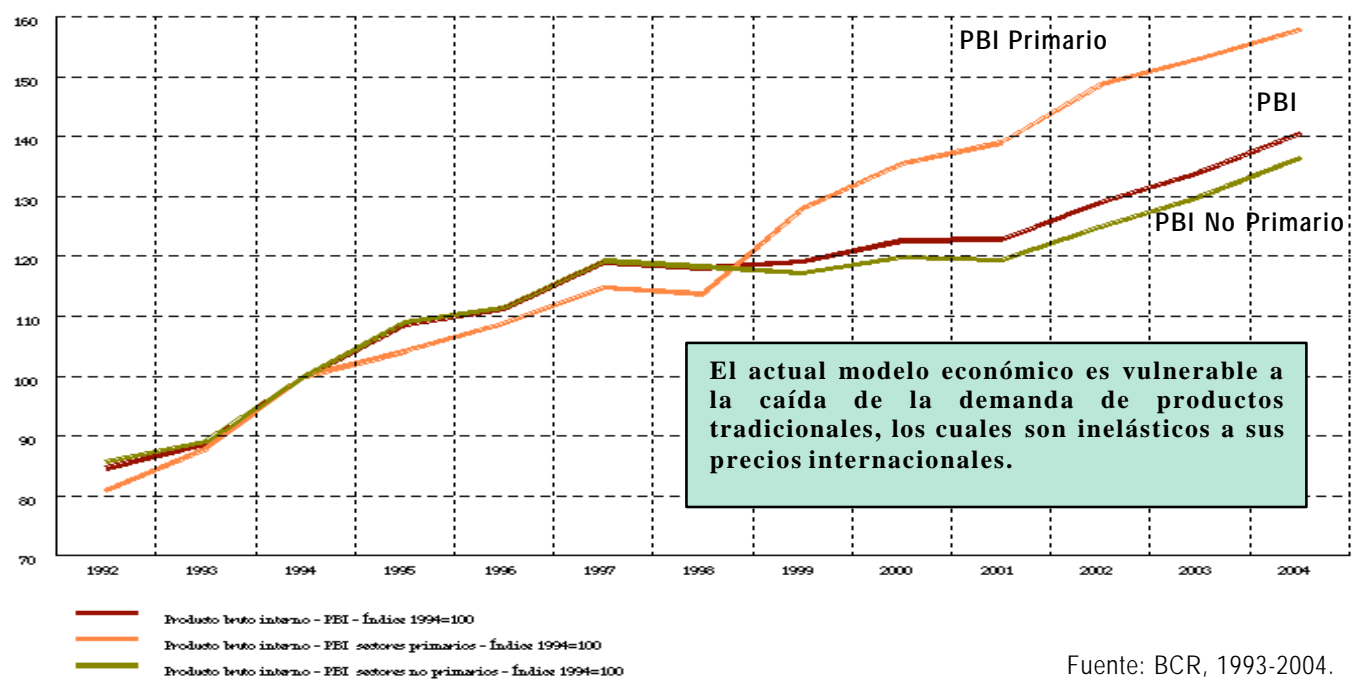

Figura 10. Evolución Indice: PBI Nacional, Sector Primario (materias primas) y Sector No Primario (industriales)

to sostenido de las exportaciones desde 1993. En promedio, el $70 \%$ de las exportaciones peruanas corresponde a productos primarios (minerales, productos agrícolas, etc.), de las cuales el $75 \%$ representa la exportación de minerales.

Con relación al intercambio comercial, el PNUD (9) menciona que los países latinoamericanos que venden principalmente productos agrícolas y minerales también se están quedando atrás, respecto a los países asiáticos, enfatizando que "... el éxito en el comercio mundial depende, cada vez, más de la capacidad de vender productos manufacturados de mayor valor agregado".

Según los datos del PNUD, en ALC los productos primarios representan por encima del $70 \%$ de las exportaciones totales. Comparativamente, los productos primarios representan apenas el $10 \%$ de los países denominados "Tigres de Asia". El Perú es una muestra palpable de lo que sucede en ALC; su estructura de exportación corresponde en promedio al $70 \%$ de productos tradicionales (Figura 11).

En cuanto al crecimiento de las exportaciones de manufactura nacional, cabe anotar que el auge de este concepto se debe, básicamente, al repunte sostenido en textiles y confecciones desde 1999 con el APTDEA con Estados Unidos y, desde el 2002, debido al incremento sorprendente de productos agroindustriales (Figura 12).

En resumen, existe una generalizada opinión nacional sobre el predominio de una economía peruana primaria-exportadora con bajo Valor Agregado, que es ineludible revertir.

El Banco Mundial, en su Informe sobre el Desarrollo Mundial 2005, sostiene que un clima propicio para las inversiones estimula el aumento de la productividad, mediante la creación de oportunidades e incentivos para que las empresas desarrollen, adapten y adopten mejores formas de hacer las cosas, añadiendo que mejorar el clima de inversión no sólo consiste en reducir los costos de la actividad empresarial. Los riesgos vinculados a la política constituyen la preocupación central para las empresas de los países en desarrollo.

El Banco Mundial consigna en el referido informe que la reducción de la pobreza está estrechamente vinculada al crecimiento, como es el caso de China, India, Uganda, países del Asia del Este, que a través de un proceso sostenido del PBI per cápita, han logrado disminuir el porcentaje de la población que vive en situación de pobreza extrema (menos de un dólar por día)

En otro estudio del Banco Mundial (1), se determinó una correlación del ingreso per cápita con el ahorro interno en el periodo 1980-1993, encontrándose un promedio mundial en el año de 1994 de $22 \%$ ahorro interno / PBI; destacando los países del Asia, que registraban valores por encima del promedio. El Perú presenta problemas estructurales para incrementar el ahorro interno: Familias mayormente pobres, empresas en problemas que requieren modernizarse y un Gobierno en déficit permanente, pero que tiende a manejar la estabilización de la economía. 


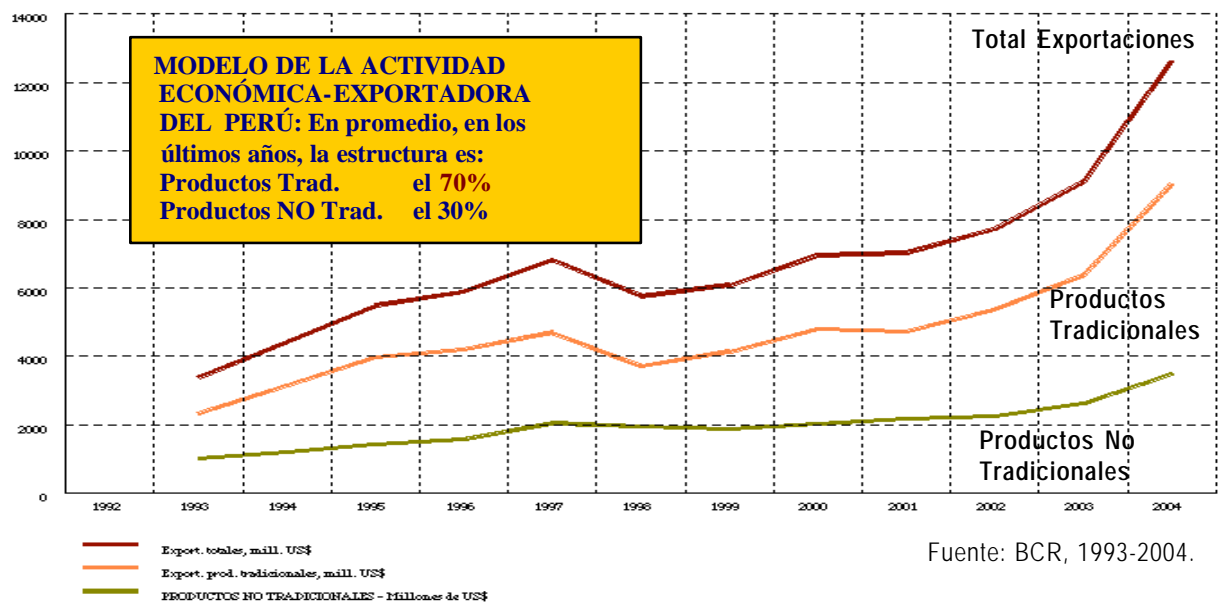

Figura 11. Estructura de Exportación: Productos Tradicionales y productos No Tradicionales

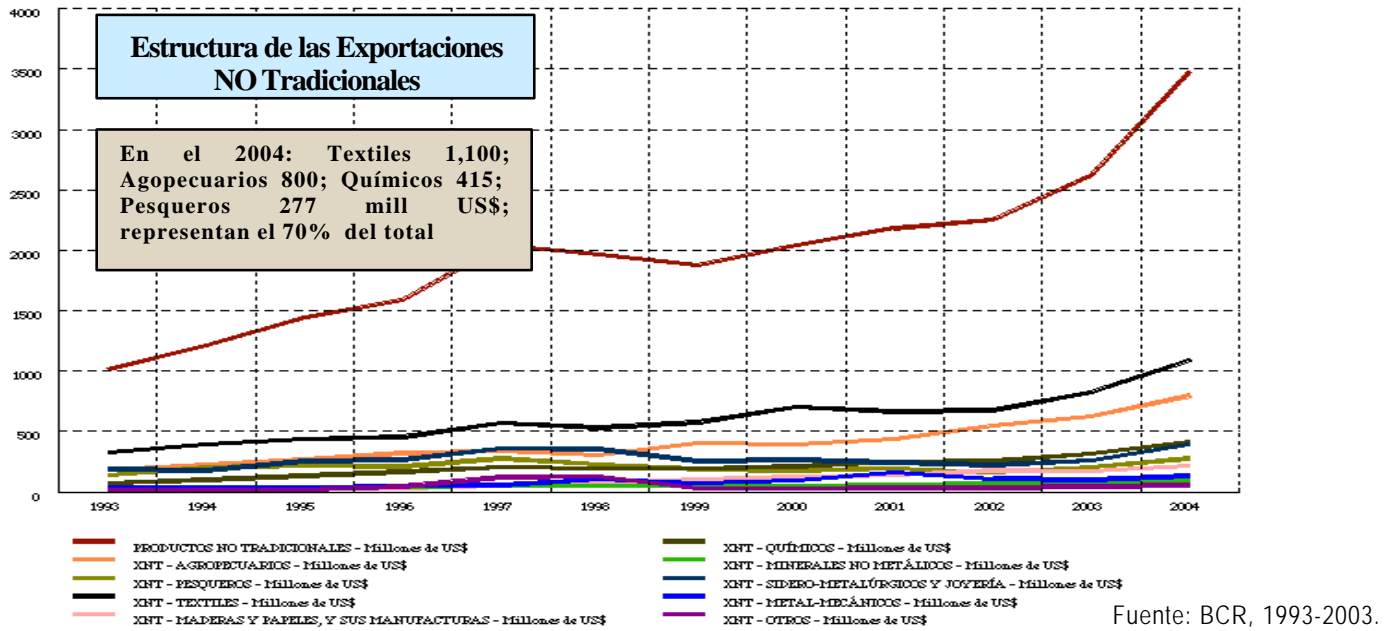

Figura 12. Evolución de las exportaciones industriales ( en millones de USD)

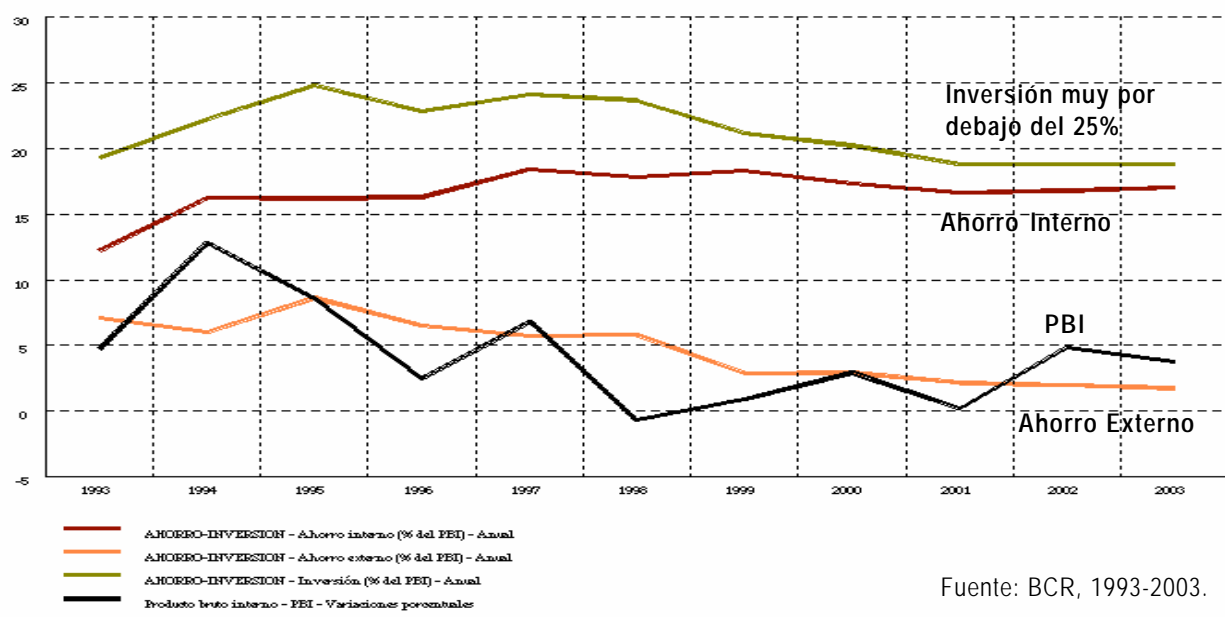

Figura 13. Perú: Evolución del PBI, Inversión, Ahorro Interno, Ahorro Externo (Variación \% del PBI) 
Por otro lado, CEPAL, el BID y otras instituciones de desarrollo instan que países como el Perú, deben crecer en $7 \%$ para resolver el problema del empleo, del sub-empleo, para lo cual recomiendan realizar los esfuerzos para contar con una relación por encima del 25\% inversión/PBI, que ha sido asumido por el Acuerdo Nacional, pero continúa sólo en "el papel".

Visto el desempeño de los países latinoamericanos, con relación a los obtenidos por ejemplo, por las economías "Tigres del Asia", el BID en su informe 2001 insta a ALC a adoptar medidas contundentes para incrementar la productividad y competitividad. Asimismo, el BID indica: "El ritmo de crecimiento del ingreso es tan lento en AL que se requeriría cerca de UN SIGLO para que la región pudiera alcanzar los niveles similares de los países desarrollados".

Pese a un buen cuadro macroeconómico del Perú, estos resultados no son suficientes para consolidar una estrategia de lucha contra la pobreza. En efecto, la relación porcentual inversión/PBI desciende a partir de 1995 (Figura 13), mostrando cifras preocupantes en los últimos cuatro años, como inferiores al $20 \%$. En la medida que no se vislumbra un cambio radical en este concepto, podemos afirmar, que contamos con: ¡Un modelo productivo que no promueve el crecimiento!

\section{¿Qué explica la gran diferencia entre los países Industriales y el mundo en desarrollo?}

Es la productividad. Según la OIT (7), hoy la productividad laboral en América Latina $(A L)$ es similar a la de 1980, consignando, que a menos que se corrijan los factores que impiden una mayor productividad de las empresas y los países, las posibilidades de progreso de la región continuarán similares a la década del 80.

De acuerdo con las cifras de la OIT, en AL un trabajador genera un nivel de producción cercano a 10,500 dólares anuales, mientras que en Estados Unidos, Japón y Europa la producción está organizada, de manera que el producto por trabajador supera los 40,000 y hasta los 50,000 dólares.

El indicador más utilizado para reflejar el crecimiento económico de un país --explica el estudio de la OIT-es el producto por habitante, que guarda una estrecha relación con la productividad laboral. En América latina, el producto por habitante, en el 2002, se aproximó a los 3900 dólares constantes de 1995, mientras que en Estados Unidos fue casi 10 veces ese valor. "El nivel tan bajo del producto por habitante --constata el informe-- refleja el rezago regional, en términos de desarrollo económico".
En ese sentido, en las dos décadas pasadas, NO se cerró la brecha de Productividad entre los países de AL -incluyendo el Perú- con los países de Asia del Este y el mundo desarrollado.

Según el BID, una economía es más competitiva si logra crecimiento sostenido de 2 parámetros: Productividad e ingreso per-cápita; pero según se desprende del análisis, para ALC, la meta de crecimiento sostenida es una META difícil de alcanzar y, por lo tanto, las perspectivas de acortar la brecha con los países desarrollados parece muy lejana.

\section{¿Cómo se explica la brecha de} productividad?

La explicación fundamental: Existencia de una profunda brecha tecnológica. Piñón (10) señala que las actividades científicas y tecnológicas van de la mano con la evolución y progreso de las sociedades, ya que transforman de manera excepcional el aparato productivo; poniendo de relieve que, la mayoría de países en desarrollo, no transitaron un camino similar al de Europa, Estados Unidos y el Japón. Perú ALC se encuentra a la zaga, en este esfuerzo de construcción del conocimiento y la innovación (Figura 14).

\section{CONCLUSIONES Y RECOMENDACIONES}

Perú, debido a su bajo desarrollo económico, es un país socialmente deprimido: Enorme pobreza y desigualdad social. Las fórmulas neoliberales aplicadas en el Perú como en ALC para combatir la pobreza y la desigualdad social, basado en el goteo de la prosperidad de la mano invisible del mercado, han fracasado.

Existen peligros inminentes:

- Que se acabe la "fiesta de las exportaciones de minerales", que ha impulsado el incremento de las divisas y del PBI.

- Aumento exponencial de la delincuencia, convirtiéndose en "una ocupación laboral".

- Que se produzca un desborde social.

Hay crisis de esperanza. Sin invertir en el conocimiento y en actividades productivas, $\mathrm{NO}$ tenemos alternativas esperanzadoras para revitalizar nuestro nivel de COMPETITIVIDAD.

Sin embargo, tenemos posibilidades: Aprovechar nuestras fortalezas. Contamos con:

1. Gente habilidosa para impulsar las PYMES.

2. Una frontera agrícola posible de sustentar polos de desarrollo agroindustriales.

3. Una gama de minerales (a diferencia de Chile, sólo cobre) posibles de convertirse en productos con mayor valor agregado. 


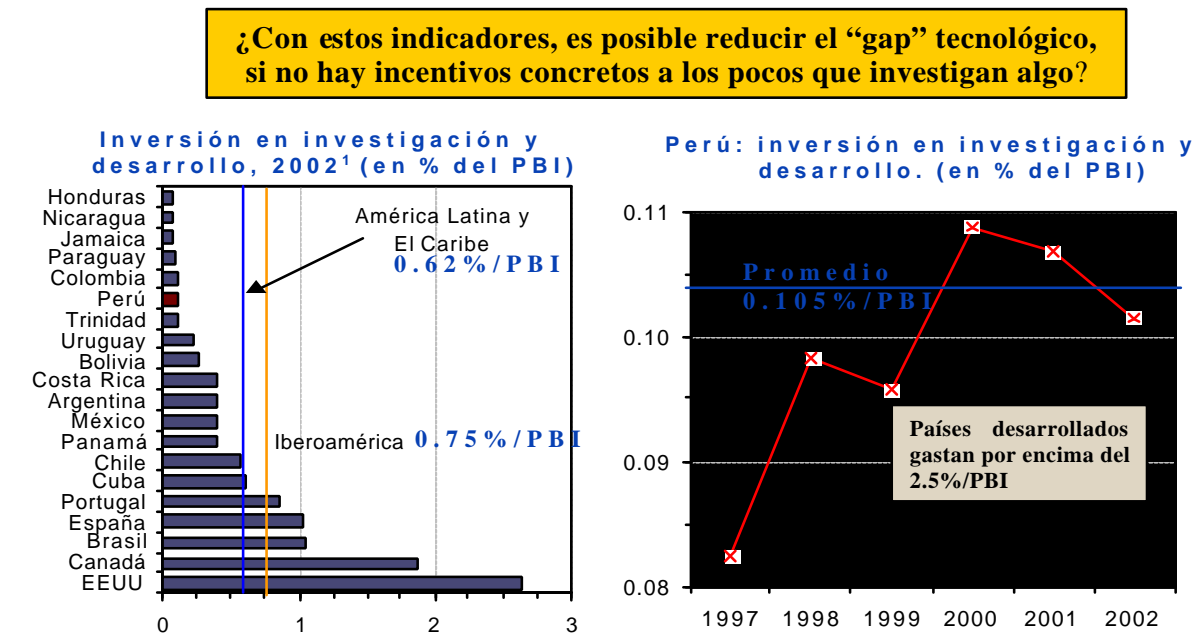

Fuente: Red Iberoamericana de Indicadores de Ciencia y Tecnología (2002) e IPE. Elaborado por IPE, Agosto 2004 ${ }^{1}$ Brasil, Costa Rica y Honduras: 2000; Portugal, Chile, Panamá, México y Trinidad \& Tobago: 2001.

Figura 14. Inversión en I+D

4. Una reserva expectante de gas natural (Gas de Camisea), que proporciona mayores posibilidades para impulsar un proceso de industrialización con la petroquímica (No perdamos la oportunidad que se presentó con el petróleo, cuando se le dio prioridad a su exportación, por el aspecto financiero), en la medida que su uso sólo en viviendas, automotriz, reconversión industrial con otros combustibles más caros y su exportación, no garantiza el impulso industrial.

A grandes desafíos, corresponden medidas radicales:

1. Impulsar decididamente las inversiones que sustenten un crecimiento del $7 \%$ del PBI.

2. Construir un marco legal de incentivos para rentabilizar las inversiones.

Sugerencias en el campo industrial:

1. Política de promoción e incentivos de las PYMES para sustentar la generación de empleo en el corto y largo plazo.

2. Construir el enlace Universidad-Estado-Empresa, sobre la base de incentivos tributarios a las empresas, para el aumento en gastos de l+D, con mecanismos para desarrollar investigación aplicada.

3. Establecer una política diferenciada de promoción de inversiones CON INCENTIVOS para atraer grandes inversiones orientadas a la exportación, que permitan el desarrollo tecnológico y aprovechamiento de nuestras materias primas para darle mayor valor agregado en tres ejes industriales:

a. Transformación Agro-Industrial

b. Industrialización de los metales (creación de la industria: Química de los metales) c. Industrialización del gas natural (en el largo plazo: Creación de una industria petroquímica), como una alternativa de mayor prioridad a la de abastecer este combustible a terceros países.

Realizando un balance de los modelos productivos y metas a plantearse al 2015, es necesario hacer un alto y volver a ordenarnos para plantear una estrategia agresiva con un cambio radical del actual modelo productivo, que considere el logro de metas mínimas sostenibles en el largo plazo:

\begin{tabular}{|l|c|l|}
\hline Variables & $\begin{array}{c}\text { Resultados Perú } \\
\mathbf{2 0 0 3 - 2 0 0 4}\end{array}$ & \multicolumn{1}{|c|}{$\begin{array}{c}\text { Metas Referenciales } \\
\mathbf{2 0 1 5}\end{array}$} \\
\hline Crecimiento PBI & $4 \%$ & $7 \%$ países emergentes \\
\hline Inversión/PBI & $18 \%$ & $20 \%-25 \%$ países emergentes \\
\hline Exportaciones /PBI & $16 \%$ & $23 \%$ mundial \\
\hline Bienes Primarios/PBI & $70 \%$ & $30 \%$ mundial \\
\hline Bienes Industriales/PBI & $30 \%$ & $70 \%$ mundial \\
\hline Inversión I\&D/PBI & $0,10 \%$ & $0,7 \%$ AL y Caribe \\
\hline Pobreza: \% población & $52 \%$ & $2, \%$ Chile \\
\hline Productividad: PBI/PEA(US\$/persona) & 900 & 12600 Chile \\
\hline
\end{tabular}

\section{BIBLIOGRAFÍA}

1. Banco Mundial (1996). FMI: Estadísticas Financieras Internacionales.

2. El Comercio. (2004). Sección Economía y Negocios. La receta de Porter para la competitividad de Latinoamérica. p. b5. Edición del 10 de junio del 2004.

3. Instituto Peruano de Economía - IPE. (2004). Ponencia "Integrando el Perú al Mundo" en el PAD de 
la Universidad de Piura del 10 de Agosto del 2004.

4. INEI-Perú. Anuncio del Jefe del INEI.p. 13. Diario La República del 25.10.05

5. MEF-Perú (2005). Marco Macroeconómico Multianual 2006-2008 (Revisado), aprobado por el Consejo de Ministros el 22.08.05; FMI (Octubre 2005) datos del mundo y ALC.

6. ONUDI (2004), Organización de las Naciones Unidas para el Desarrollo Industrial. Informe sobre el Desarrollo Industrial 2004. Julio 2004.

7. Oficina Regional para América Latina y El Caribe, OIT. (2004). Panorama Laboral. Lima, Perú.

8. Porlles, J.(2004). Competitividad: Estructura Industrial del Perú y Perspectivas. Revista de la Sociedad Química del Perú. Vol 70., Nro 4: p 236. Octubre-Diciembre 2004
9. Programa de la Naciones Unidas para el DesarroIlo - PNUD. (2005). El Informe del Desarrollo Humano. Lima, Perú.

10. Pinón, F. (2004). Ciencia y Tecnología en América Latina: Una posibilidad para el desarrollo. Secretaría General de la Organización de Estados Iberoamericanos para la Educación y la Cultura (OEI). Madrid, España.

11.Singh, A. (2005). El Contexto Mundial y las Perspectivas Regionales para América Latina y El Caribe. Departamento del Hemisferio Occidental, FMI. Octubre 2005.

12. UNCTAD. (2005). Conferencia de las Naciones Unidas sobre Comercio y Desarrollo. World Investment Report 2005. Septiembre 2005.

13.World Economic Forum - WEF. (2006). Global Competitiveness Report 2005-2006. 